\title{
Maximal regularity for a class of integro-differential equations with infinite delay in Banach spaces
}

\author{
by
}

\section{Valentin Keyantuo (Puerto Rico) and Carlos Lizama (Santiago)}

\begin{abstract}
We use Fourier multiplier theorems to establish maximal regularity results for a class of integro-differential equations with infinite delay in Banach spaces. Concrete equations of this type arise in viscoelasticity theory. Results are obtained for periodic solutions in the vector-valued Lebesgue and Besov spaces. An application to semilinear equations is considered.
\end{abstract}

1. Introduction. Initiated by L. Weis in [32] (see also [31]), the use of operator-valued Fourier multipliers in the investigation of maximal regularity for abstract differential equations has been very successful of late. For some recent papers on the subject, we refer to [3], [5], [2] and the references cited there. Maximal regularity has proven very useful in handling some concrete nonlinear evolution equations, as evidenced by the papers [2] and [24] which deal with the Navier-Stokes equations of fluid dynamics. For the use of maximal regularity in integro-differential equations, we mention among others the works of Da Prato and Clément [11], Sforza [28] and Prüss's monograph [26].

In the present article, we consider the following integro-differential equation with infinite delay:

$$
\begin{aligned}
\gamma_{0} u^{\prime}(t)+\frac{d}{d t}\left(\int_{-\infty}^{t} b(t-s) u(s) d s\right)+\gamma_{\infty} u(t) & \\
& =c_{0} A u(t)-\int_{-\infty}^{t} a(t-s) A u(s) d s+f(t), \quad t \in \mathbb{R} .
\end{aligned}
$$

Here, $\gamma_{0}, \gamma_{\infty}, c_{0}$ are constants and $a(\cdot), b(\cdot) \in L^{1}\left(\mathbb{R}_{+}\right)$. Equations of the form (1.1) appear in a variety of applied problems. They typically arise in

2000 Mathematics Subject Classification: Primary 45N05, 35K90; Secondary 45K05, 45D05, 46N20.

The first author is supported in part by Convenio de Cooperación Internacional (CONICYT) grant \#7010675 and the second author is partially financed by FONDECYT grant \#1010675. 
mathematical physics by some constitutive laws pertaining to materials with memory when combined with the usual conservation laws such as balance of energy or balance of momentum. For details concerning the underlying physical principles, we refer to Coleman-Gurtin [13], Lunardi [23], Nunziato [25], and Prüss [26] (particularly Chapter II, Section 9).

We study equation (1.1) in various spaces of $2 \pi$-periodic vector-valued functions: $L^{p}(0,2 \pi ; X), C^{\alpha}(0,2 \pi ; X)$ (Hölder spaces), $B_{p q}^{s}(0,2 \pi ; X)$ (Besov spaces) where the parameters satisfy $1 \leq p, q \leq \infty, s \in \mathbb{R}, 0<\alpha<1$. Here $X$ is a Banach space, $A: D(A) \subset X \rightarrow X$ is a (not necessarily densely defined) closed linear operator and $f$ is an $X$-valued function defined on $[0,2 \pi]$.

Hölder-continuous solutions for the full equation (1.1) have been studied on the real line by Lunardi [23] in the case where $A$ is, e.g., the Laplace operator in a bounded domain $\Omega \subset \mathbb{R}^{N}$ and the Banach space is $X=$ $C(\bar{\Omega})$. Da Prato and Lunardi [14] investigated periodic solutions of equation $(1.1)$ in the case $b(\cdot) \equiv 0$ (see also Da Prato-Lunardi [15]). Clément and Da Prato [11] studied equation (1.1) on the real line in the case where $a(\cdot) \equiv 0$ and obtained maximal regularity results in Sobolev and Hölder spaces as well as the space of bounded uniformly continuous functions. The results of [11] were then used by Sforza [28] to derive global existence and uniqueness results for the associated semilinear problem. We note that their results do not include the Lebesgue scale $L^{p}$ (except when $p=2$, in which case Plancherel's theorem is available). A key assumption in all the abovementioned works is that $A$ generates an analytic semigroup (not necessarily strongly continuous). However, they also treat more general operator-valued kernels.

Our results use, on the one hand, recent papers by Arendt-Bu [5], [6], Arendt-Batty- $\mathrm{Bu}[3]$ where the above problem is studied with $a(\cdot) \equiv 0$, $b(\cdot) \equiv 0$ and, on the other hand, Keyantuo-Lizama [20] where the problem (1.1) is studied in the case $b(\cdot) \equiv 0$ and $\gamma_{\infty}=0$. One remarkable fact is that in the context of resolvents of closed linear operators in Banach spaces, $L^{p}$-multipliers can be completely characterized. See for example [5, Theorem 2.3] and [6, Theorem 4.1] for a precise formulation of this result. The fact that one obtains a complete characterization of well-posedness contrasts with the earlier approaches mentioned above.

We obtain maximal regularity in all the above spaces: Lebesgue-Bochner, Hölder and Besov spaces. In the case of Hölder spaces, which was considered by Da Prato-Lunardi [14, Theorem 2.3], [15, Theorem 4.3], the result obtained is a complete and very simple characterization of maximal regularity. The conditions that we impose on the kernel $a(\cdot)$ are satisfied by a large class of functions, including the cases $a(t)=\sum_{j=1}^{n} c_{j} e^{-\alpha_{j} t}, b(t)=\sum_{j=1}^{m} d_{j} e^{-\beta_{j} t}$ 
where $\alpha_{j}, \beta_{j}, c_{j}, d_{j}$ are positive real numbers and $n, m \in \mathbb{N}$. These correspond to the most common kernels encountered in the applications.

In contrast with the above papers on the subject our assumptions are weaker. We do not make any parabolicity assumption on the operator $A$, not even that $A$ generates a semigroup. Moreover, we obtain the results in all the spaces indicated in the first paragraph above.

The paper is organized as follows: in Section 2, we prove a general maximal regularity result for $(1.1)$ in the Lebesgue-Bochner spaces $L^{p}(0,2 \pi ; X)$ and the Besov spaces $B_{p q}^{s}(0,2 \pi ; X)$ (in particular the space $C^{\alpha}(0,2 \pi ; X)$ ) of Hölder-continuous functions in terms of operator-valued Fourier multipliers. In Section 3, we give conditions on the data ensuring applicability of the result established in Section 2. In the case of $L^{p}(0,2 \pi ; X), 1<p<\infty$, the result involves UMD-spaces and $R$-boundedness as well as a condition on the resolvent of $A$. In the other cases, the theorems involve only boundedness of the resolvent and are therefore suitable for the applications. In the last section, we apply the results to a semilinear problem.

2. A general maximal regularity result. In this section, we establish a general maximal regularity result for periodic solutions of equation (1.1) in the vector-valued Lebesgue and Besov spaces. The result is in terms of operator-valued Fourier multipliers. Thus, we consider the problem

$$
\left\{\begin{array}{l}
\gamma_{0} u^{\prime}(t)+\frac{d}{d t}\left(\int_{-\infty}^{t} b(t-s) u(s) d s\right)+\gamma_{\infty} u(t) \\
=c_{0} A u(t)-\int_{-\infty}^{t} a(t-s) A u(s) d s+f(t), \quad 0 \leq t \leq 2 \pi, \\
u(0)=u(2 \pi) .
\end{array}\right.
$$

For a function $f \in L^{1}(0,2 \pi ; X)$, we denote by $\widehat{f}(k), k \in \mathbb{Z}$, the $k$ th Fourier coefficient of $f$ :

$$
\widehat{f}(k)=\frac{1}{2 \pi} \int_{0}^{2 \pi} e_{-k}(t) f(t) d t,
$$

where $e_{k}(t)=e^{i k t}, t \in \mathbb{R}$.

Let $u \in L^{1}((0,2 \pi) ; X)$. We denote again by $u$ the periodic extension to $\mathbb{R}$. Let $a \in L^{1}\left(\mathbb{R}_{+}\right)$. We first observe from

$$
F(t):=\int_{-\infty}^{t} a(t-s) u(s) d s=\int_{0}^{\infty} a(s) u(t-s) d s
$$

that $F$ is bounded and periodic of period $T=2 \pi$ as $u$. Now using Fubini's 
theorem and (2.2) we obtain, for $k \in \mathbb{Z}$,

$$
\begin{aligned}
\widehat{F}(i k) & =\frac{1}{2 \pi} \int_{0}^{2 \pi} e^{-i k t} F(t) d t=\frac{1}{2 \pi} \int_{0}^{2 \pi} e^{-i k t} \int_{0}^{\infty} a(s) u(t-s) d s d t \\
& =\frac{1}{2 \pi} \int_{0}^{2 \pi} e^{-i k(t-s)} \int_{0}^{\infty} e^{-i k s} a(s) u(t-s) d s d t \\
& =\frac{1}{2 \pi} \int_{0}^{2 \pi} e^{-i k(t-s)} u(t-s) d t \int_{0}^{\infty} e^{-i k s} a(s) d s
\end{aligned}
$$

and hence

$$
\widehat{F}(k)=\widetilde{a}(i k) \widehat{u}(k), \quad k \in \mathbb{Z},
$$

where $\widetilde{a}(\lambda)=\int_{0}^{\infty} e^{-\lambda t} a(t) d t$ denotes the Laplace transform of $a$. This is a key identity in our investigations.

Let $X, Y$ be Banach spaces. We denote by $\mathcal{L}(X, Y)$ the set of all bounded linear operators from $X$ to $Y$. When $X=Y$, we simply write $\mathcal{L}(X)$. The identity operator on $X$ will be denoted by $I$. For a linear operator $A$ on $X$ with domain $D(A)$, we denote its resolvent set by $\varrho(A)$.

For results about operator-valued Fourier multipliers and $R$-boundedness (which we use in the next section), we refer to Amann [1], Bourgain [7], Clément-de Pagter-Sukochev-Witvliet [12], Weis [31, 32], Girardi-Weis [18], [19], Clément-Da Prato [11] and Arendt-Bu [5]. Here, we will merely recall the appropriate definitions.

We shall frequently identify the spaces of (vector- or operator-valued) functions defined on $[0,2 \pi]$ with their periodic extensions to $\mathbb{R}$. Thus, in this section, we consider the space $L_{2 \pi}^{p}(\mathbb{R} ; X)$ (denoted also $L^{p}(0,2 \pi ; X)$, $1 \leq p \leq \infty)$ of all $2 \pi$-periodic Bochner measurable $X$-valued functions $f$ such that the restriction of $f$ to $[0,2 \pi]$ is $p$-integrable.

Definition 2.1. For $1 \leq p \leq \infty$, we say that a sequence $\left\{M_{k}\right\}_{k \in \mathbb{Z}} \subset$ $\mathcal{L}(X, Y)$ is an $L^{p}$-multiplier if for each $f \in L^{p}(0,2 \pi ; X)$ there exists $u \in$ $L^{p}(0,2 \pi ; Y)$ such that

$$
\widehat{u}(k)=M_{k} \widehat{f}(k) \quad \text { for all } k \in \mathbb{Z} .
$$

In the case of Besov spaces (see e.g. [6], [10], [30, p. 195]), we have the following.

Definition 2.2. Let $X$ and $Y$ be Banach spaces and let $\left\{M_{k}\right\}_{k \in \mathbb{Z}} \subset$ $\mathcal{L}(X, Y)$. We will say that $\left\{M_{k}\right\}_{k \in \mathbb{Z}}$ is a $B_{p, q}^{s}$-multiplier if for each $f \in$ $B_{p, q}^{s}(0,2 \pi ; X)$ there exists $u \in B_{p, q}^{s}(0,2 \pi ; Y)$ such that

$$
\widehat{u}(k)=M_{k} \widehat{f}(k) \quad \text { for all } k \in \mathbb{Z} .
$$


From the uniqueness theorem of Fourier series, it follows that $u$ is uniquely determined by $f$.

REMARK 2.3. It is clear from the definitions that if $\left\{M_{k}\right\}_{k \in \mathbb{Z}} \subset \mathcal{L}(X, Y)$ and $\left\{N_{k}\right\}_{k \in \mathbb{Z}} \subset \mathcal{L}(Y, Z)$ are Fourier multipliers then $\left\{N_{k} M_{k}\right\}_{k \in \mathbb{Z}} \subset \mathcal{L}(X, Z)$ is a Fourier multiplier as well.

We note that periodic vector-valued Besov spaces were only introduced recently (see the paper [6] where Fourier multipliers in this context are studied for the first time), in contrast with periodic Hölder spaces which had been used in the literature previously. Periodic Besov spaces in the scalar case, also known as Lipschitz spaces, are much older (see e.g. [10, Chapter 4] and [30] and the references cited there).

We denote by $\mathcal{Y}=\mathcal{Y}(X)$ any of the following spaces of $X$-valued functions: $L^{p}(0,2 \pi ; X), 1 \leq p \leq \infty, B_{p, q}^{s}(0,2 \pi ; X), 1 \leq p, q \leq \infty, s>0$. Moreover we define

$$
\mathcal{Y}^{[1]}=\left\{u \in \mathcal{Y}: u \text { is almost everywhere differentiable and } u^{\prime} \in \mathcal{Y}\right\} .
$$

REMARK 2.4. If $u \in \mathcal{Y}^{[1]}$, then $u$ is bounded. In the case where $\mathcal{Y}=$ $L^{p}(0,2 \pi ; X), 1 \leq p \leq \infty$, this follows from [5, Lemma 2.1] and Hölder's inequality. When $\mathcal{Y}=B_{p, q}^{s}(0,2 \pi ; X), 1 \leq p, q \leq \infty, s>0$, this is a consequence of [6, Theorem 1.3 ((i) and (iv))] and Hölder's inequality.

Recall that we denote by $\widetilde{g}(\lambda)=\int_{0}^{\infty} e^{-\lambda t} g(t) d t$ the Laplace transform of the function $g$. Henceforth, for $g \in L^{1}\left(\mathbb{R}_{+}\right)$, set

$$
g_{k}=\widetilde{g}(i k), \quad k \in \mathbb{Z} .
$$

We consider the following two hypotheses:

(H0a) $g \in L^{1}\left(\mathbb{R}_{+}\right)$and the sequence $\left(g_{k} I\right)_{k \in \mathbb{Z}} \subset \mathcal{L}(X)$ is a $\mathcal{Y}$-multiplier. (H0b) $\quad c_{0}-g_{k} \neq 0$ for all $k \in \mathbb{Z}$ and the sequence $\left(\left(c_{0}-g_{k}\right)^{-1} I\right)_{k \in \mathbb{Z}} \subset$ $\mathcal{L}(X)$ is a $\mathcal{Y}$-multiplier.

We shall write $(\mathrm{H} 0)$ when $(\mathrm{HOa})$ and $(\mathrm{HOb})$ are both satisfied (or required). We shall also frequently write $\left(a_{k}\right)$ when referring to $\left(a_{k} I\right)$ as operators on $X$.

REMARK 2.5. Let $g \in L^{1}\left(\mathbb{R}_{+}\right)$. We denote again by $g$ its extension by 0 on $(-\infty, 0]$. Then the Fourier transform of $g$ is a multiplier of $L^{p}(\mathbb{R}), 1 \leq$ $p \leq \infty$. In fact, it is well known that the multipliers of $L^{1}(\mathbb{R})$ are the Fourier transforms of bounded measures.

Since in this case the Fourier transform of $g$ is a continuous function, it follows from [29, Chap. VII, Theorem 3.8] (by using periodization) that at least when $X=\mathbb{C}$ (or when $X$ is a Hilbert space), the sequence $\left(g_{k}\right)$ is an $L^{p}$-multiplier for $1 \leq p \leq \infty$. 
We denote by $H_{\text {per }}^{1}(0,2 \pi ; X)$ the space of all $u \in L^{p}(0,2 \pi ; X)$ for which there exists $v \in L^{p}(0,2 \pi ; X)$ such that $\widehat{v}(k)=i k \widehat{u}(k)$ for all $k \in \mathbb{Z}$.

Definition 2.6. Let $1<p<\infty$. A function $u \in H_{\mathrm{per}}^{1}(0,2 \pi ; X)$ is called a strong $L^{p}$-solution of (2.1) if $u(t) \in D(A)$ and equation (2.1) holds for almost all $t \in[0,2 \pi]$.

For Besov spaces, we adopt the following definition of solutions.

Definition 2.7. Let $1 \leq p, q \leq \infty$ and $s>0$. A function $u \in$ $B_{p, q}^{s+1}(0,2 \pi ; X)$ is called a strong $B_{p, q}^{s}$-solution of $(2.1)$ if $u(t) \in D(A)$ and (2.1) holds for $t \in[0,2 \pi]$.

REMARK 2.8. By [6, Theorem 1.3], if $u$ is a strong $B_{p, q}^{s}$-solution of (2.1), then $u$ is differentiable a.e. and $u^{\prime} \in B_{p, q}^{s}(0,2 \pi ; X)$. This follows from $u \in$ $B_{p, q}^{s+1}(0,2 \pi ; X)$. Moreover, in the $L^{p}$ case, $\mathcal{Y}^{[1]}=H_{\text {per }}^{1}(0,2 \pi ; X)$, and in the $B_{p q}^{s}$ case, $s>0, \mathcal{Y}^{[1]}=B_{p q}^{s+1}(0,2 \pi ; X)$.

For $k \in \mathbb{Z}$ and $a_{k} \neq c_{0}$ we let

$$
\begin{aligned}
d_{k} & :=\frac{i k\left(\gamma_{0}+b_{k}\right)+\gamma_{\infty}}{c_{0}-a_{k}}, \\
M_{k} & :=\frac{i k}{c_{0}-a_{k}}\left(d_{k} I-A\right)^{-1}, \quad k \in \mathbb{Z} .
\end{aligned}
$$

In what follows, we denote by $a \dot{*} u$ the "convolution" of $a$ and $u$ defined by

$$
(a \dot{*} b)(t):=\int_{0}^{\infty} a(s) u(t-s) d s=\int_{-\infty}^{t} a(t-s) u(s) d s,
$$

where $a \in L^{1}\left(\mathbb{R}_{+}\right)$and $u:[0,2 \pi] \rightarrow X$ is extended by periodicity to $\mathbb{R}$, the same notation being kept for the extension. The following is the main result of this section.

Theorem 2.9. Let $X$ be a Banach space and $A: D(A) \subset X \rightarrow X$ be a closed linear operator. Assume that the function a $(\cdot)$ satisfies (H0) and $b(\cdot)$ satisfies (H0a). Then the following assertions are equivalent for $1<p<\infty$ :

(i) For every $f \in \mathcal{Y}$ there exists a unique strong $\mathcal{Y}$-solution of (2.1).

(ii) $\left\{d_{k}\right\}_{k \in \mathbb{Z}} \subseteq \varrho(A)$ and $\left(M_{k}\right)_{k \in \mathbb{Z}}$ is a $\mathcal{Y}$-multiplier.

Moreover if (ii) is satisfied, then the following maximal regularity property holds: $u^{\prime}, A u, b \dot{*} u, \frac{d}{d t}(b \dot{*} u), a \dot{*} A u \in \mathcal{Y}$. Furthermore, there exists a constant $C>0$ independent of $f \in \mathcal{Y}$ such that

$$
\left\|u^{\prime}\right\|_{\mathcal{Y}}+\|A u\|_{\mathcal{Y}}+\|a \dot{*} A u\|_{\mathcal{Y}}+\|b \dot{*} u\|_{\mathcal{Y}}+\left\|\frac{d}{d t}(b \dot{*} u)\right\|_{\mathcal{Y}} \leq C\|f\|_{\mathcal{Y}}
$$


Proof. (i) $\Rightarrow$ (ii). Let $k \in \mathbb{Z}$ and $y \in X$. Define $f(t)=e^{i k t} y$. Then $\widehat{f}(k)=y$. By assumption, there exists $u \in \mathcal{Y}^{[1]}$ such that

$$
\frac{d}{d t}\left(\gamma_{0} u(t)+b \dot{*} u\right)+\gamma_{\infty} u(t)=c_{0} A u(t)-\int_{-\infty}^{t} a(t-s) A u(s) d s+f(t) .
$$

Taking Fourier series on both sides of (2.1) and using (2.3) and the closedness of $A$, we deduce that $\widehat{u}(k) \in D(A)$ and $i k\left(\gamma_{0}+\widetilde{b}(i k)\right) \widehat{u}(k)+\gamma_{\infty} \widehat{u}(k)=$ $c_{0} A \widehat{u}(k)-\widetilde{a}(i k) A \widehat{u}(k)+\widehat{f}(k)=A\left(c_{0}-\widetilde{a}(i k)\right) \widehat{u}(k)+y$. In view of the notations adopted above (see (2.5)), we therefore have

$$
\left(i k\left(\gamma_{0}+b_{k}\right)+\gamma_{\infty}\right) \widehat{u}(k)-A\left(c_{0}-a_{k}\right) \widehat{u}(k)=y .
$$

Thus, $i k\left(\gamma_{0}+b_{k}\right) I+\gamma_{\infty} I-\left(c_{0}-a_{k}\right) A$ is surjective. Next we prove that $i k\left(\gamma_{0}+b_{k}\right) I+\gamma_{\infty} I-\left(c_{0}-a_{k}\right) A$ is one-to-one. Let $x \in D(A)$. If $\left(i k\left(\gamma_{0}+b_{k}\right) I+\gamma_{\infty} I-\left(c_{0}-a_{k}\right) A\right) x=0$, then

$$
A x=\frac{i k\left(\gamma_{0}+b_{k}\right)+\gamma_{\infty}}{c_{0}-a_{k}} x=d_{k} x,
$$

and hence $u(t):=e^{i k t} x$ defines a periodic solution of $\frac{d}{d t}\left(\gamma_{0} u(t)+b \dot{*} u\right)+$ $\gamma_{\infty} u(t)=c_{0} A u(t)-\int_{-\infty}^{t} a(t-s) A u(s) d s$. In fact, note first that $(b \dot{*} u)(t)=$ $b_{k} u(t) x$, and hence

$$
\begin{aligned}
c_{0} A u(t) & -\int_{-\infty}^{t} a(t-s) A u(s) d s \\
& =c_{0} e^{i k t} A x-\int_{-\infty}^{t} a(t-s) e^{i k s} A x d s=c_{0} e^{i k t} A x-\int_{0}^{\infty} a(s) e^{i k(t-s)} A x d s \\
& =c_{0} e^{i k t} A x-e^{i k t} \int_{0}^{\infty} a(s) e^{-i k s} d s A x \\
& =c_{0} e^{i k t} A x-e^{i k t} a_{k} A x=e^{i k t}\left(c_{0}-a_{k}\right) A x \\
& =\left(i k\left(\gamma_{0}+b_{k}\right)+\gamma_{\infty}\right) e^{i k t} x=\frac{d}{d t}\left(\gamma_{0} u(t)+(b \dot{*} u)(t)\right)+\gamma_{\infty} u(t) .
\end{aligned}
$$

Hence $u=0$ by the assumption of uniqueness and therefore $x=0$. Since $A$ is closed, we conclude that $\left\{d_{k}\right\}_{k \in \mathbb{Z}} \subset \varrho(A)$.

The verification that $\left(M_{k}\right)$ is a $\mathcal{Y}$-multiplier is now simple. Indeed, if $f \in \mathcal{Y}$, there exists $u \in \mathcal{Y}^{[1]}$ solving equation (2.1). By taking Fourier series, we obtain

$$
\left(i k\left(\gamma_{0}+b_{k}\right)+\gamma_{\infty}\right) \widehat{u}(k)-A\left(c_{0}-a_{k}\right) \widehat{u}(k)=\widehat{f}(k) .
$$

Since we have proved that $i k\left(\gamma_{0}+b_{k}\right) I+\gamma_{\infty} I-\left(c_{0}-a_{k}\right) A$ is invertible, we 
may rewrite this as

$$
\begin{aligned}
\widehat{u}(k) & =\left[i k\left(\gamma_{0}+b_{k}\right) I+\gamma_{\infty} I-\left(c_{0}-a_{k}\right) A\right]^{-1} \widehat{f}(k) \\
& =\frac{1}{c_{0}-a_{k}}\left(d_{k} I-A\right)^{-1} \widehat{f}(k), \quad k \in \mathbb{Z} .
\end{aligned}
$$

Since $u \in \mathcal{Y}^{[1]}, u^{\prime} \in \mathcal{Y}$ and for $k \in \mathbb{Z}, i k \widehat{u}(k)=\left(\widehat{u}^{\prime}\right)(k)$. From this and the definitions, it follows that $\left(M_{k}\right)_{k \in \mathbb{Z}}$ is a $\mathcal{Y}$-multiplier.

(ii) $\Rightarrow$ (i). Let $f \in \mathcal{Y}$. By assumption, there exists $v \in \mathcal{Y}$ such that

$$
\widehat{v}(k)=M_{k} \widehat{f}(k), \quad k \in \mathbb{Z} .
$$

Since $M_{k}=i k\left(c_{0}-a_{k}\right)^{-1}\left(b_{k} I-A\right)^{-1}$, it follows from [5, Lemma 2.2] that if we set $N_{k}=\left(c_{0}-a_{k}\right)^{-1}\left(d_{k} I-A\right)^{-1}$, then $\left(N_{k}\right)$ is a multiplier as well. Hence there exists $u \in \mathcal{Y}$ such that $\widehat{u}(k)=N_{k} \widehat{f}(k), k \in \mathbb{Z}$. Clearly, $\widehat{u}(k) \in D(A)$ and

$$
\left(c_{0}-a_{k}\right) \widehat{u}(k)=\left(d_{k}-A\right)^{-1} \widehat{f}(k), \quad k \in \mathbb{Z} .
$$

Thus we obtain

$$
i k\left(\gamma_{0}+b_{k}\right) \widehat{u}(k)+\gamma_{\infty} \widehat{u}(k)=c_{0} A \widehat{u}(k)-a_{k} A \widehat{u}(k)+\widehat{f}(k), \quad k \in \mathbb{Z} .
$$

Note that $i k \widehat{u}(k)=i k N_{k} \widehat{f}(k)=M_{k} \widehat{f}(k)=\widehat{v}(k), k \in \mathbb{Z}$. It follows from [5] or [6] that $u(\cdot)$ is differentiable and $u^{\prime}(\cdot) \in \mathcal{Y}$. Now we show that $u(t) \in D(A)$ for a.e. $t \in[0,2 \pi]$. As already remarked, $\widehat{u}(k) \in D(A), k \in \mathbb{Z}$. We have, for $k \in \mathbb{Z}$,

$$
\begin{aligned}
A \widehat{u}(k) & =A\left[\frac{1}{c_{0}-a_{k}}\left(d_{k}-A\right)^{-1}\right] \widehat{f}(k)=\frac{1}{c_{0}-a_{k}} A\left(d_{k}-A\right)^{-1} \widehat{f}(k) \\
& =\frac{1}{c_{0}-a_{k}}\left[d_{k}\left(d_{k}-A\right)^{-1}-I\right] \widehat{f}(k) \\
& =\left[\frac{1}{c_{0}-a_{k}} d_{k}\left(d_{k}-A\right)^{-1}-\frac{1}{c_{0}-a_{k}} I\right] \widehat{f}(k) .
\end{aligned}
$$

In view of assumption (H0) on $\left(a_{k}\right)$ and assumption (H0a) on $\left(b_{k}\right)$, $\left(\left(c_{0}-a_{k}\right)^{-1} d_{k}\left(d_{k}-A\right)^{-1}-\left(c_{0}-a_{k}\right)^{-1} I\right)$ is a $\mathcal{Y}$-multiplier. In fact,

$$
\begin{aligned}
d_{k}\left(d_{k}-A\right)^{-1} & =\frac{i k\left(\gamma_{0}+b_{k}\right)+\gamma_{\infty}}{c_{0}-a_{k}}\left(d_{k}-A\right)^{-1} \\
& =\frac{i k\left(\gamma_{0}+b_{k}\right)}{c_{0}-a_{k}}\left(d_{k}-A\right)^{-1}+\frac{\gamma_{\infty}}{c_{0}-a_{k}}\left(d_{k}-A\right)^{-1} \\
& =\left(\gamma_{0}+b_{k}\right) M_{k}+\gamma_{\infty} N_{k} .
\end{aligned}
$$

Since $A$ is closed, we conclude that $u(t) \in D(A)$ for a.e. $t \in[0,2 \pi]$. Clearly, $b \dot{*} u \in \mathcal{Y}$. We also note that

$$
i k\left[b_{k} \widehat{u}(k)\right]=b_{k} M_{k} \widehat{f}(k), \quad k \in \mathbb{Z} .
$$

Since $\left(M_{k}\right)$ is a multiplier, we invoke assumption (H0a) to conclude that $\frac{d}{d t}(b \dot{*} u) \in \mathcal{Y}$. In order to prove that $u(\cdot)$ is a solution of $(2.1)$, it remains 
to show that $a \dot{*} u, a \dot{*} A u \in \mathcal{Y}$ (we actually prove more than what is needed to deduce that the integro-differential equation is satisfied). But this follows from the corresponding proof for $u(\cdot)$ and $A u(\cdot)$ and the fact that $\left(a_{k} I\right)$ is a multiplier.

From (2.3) and the uniqueness theorem of Fourier coefficients, we conclude that $u(\cdot)$ satisfies $(2.1)$ for almost all $t \in[0,2 \pi]$.

It remains to prove uniqueness. To this end, let $u$ be such that

$$
\frac{d}{d t}\left(\gamma_{0} u(t)+(b \dot{*} u)(t)\right)+\gamma_{\infty} u(t)-c_{0} A u(t)+\int_{-\infty}^{t} a(t-s) A u(s) d s=0 .
$$

Then, taking Fourier coefficients, for $k \in \mathbb{Z}$ we obtain $\widehat{u}(k) \in D(A)$ and $\left(\left(i k\left(\gamma_{0}+b_{k}\right)+\gamma_{\infty}\right) I-\left(c_{0}-a_{k}\right) A\right) \widehat{u}(k)=0$. Since

$$
d_{k}=\frac{i k\left(\gamma_{0}+b_{k}\right)+\gamma_{\infty}}{c_{0}-a_{k}} \in \varrho(A)
$$

this implies that $\widehat{u}(k)=0$ for all $k \in \mathbb{Z}$ and thus $u=0$.

The last assertion of the theorem is a direct consequence of the fact that $u^{\prime}, A u, b \dot{*} u, \frac{d}{d t}(b \dot{*} u), a \dot{*} A u \in \mathcal{Y}$ are defined through the operatorvalued Fourier multipliers $\left(i k N_{k}\right)_{k \in \mathbb{Z}},\left(\left(c_{0}-a_{k}\right)^{-1}\left[d_{k}\left(d_{k}-A\right)^{-1}-I\right]\right)_{k \in \mathbb{Z}}$, $\left(b_{k} N_{k}\right)_{k \in \mathbb{Z}},\left(i k b_{k} N_{k}\right)_{k \in \mathbb{Z}},\left(a_{k}\left(c_{0}-a_{k}\right)^{-1}\left[d_{k}\left(d_{k}-A\right)^{-1}-I\right]\right)_{k \in \mathbb{Z}}$ respectively. See e.g. [5, Proposition 1.1] for the case where $\mathcal{Y}=L^{p}(0,2 \pi ; X)$. The case $\mathcal{Y}=B_{p, q}^{s}(0,2 \pi ; X)$ can be treated in a similar way, by application of the uniqueness theorem for Fourier series along with the Closed Graph Theorem.

REMARK 2.10. Inequality (2.9) is known as the maximal regularity property for equation (2.1). From it we deduce that, for example, if $\mathcal{Y}=$ $L^{p}(0,2 \pi ; X)$, then the operator $L$ defined by

$$
\begin{aligned}
D(L)= & H_{\text {per }}^{1, p}(0,2 \pi ; X) \cap L^{p}(0,2 \pi ; D(A)) \\
(L u)(t)= & \frac{d}{d t}\left(\gamma_{0} u(t)+\int_{-\infty}^{t} b(t-s) u(s) d s\right) \\
& +\gamma_{\infty} u(t)-c_{0} A u(t)+\int_{-\infty}^{t} a(t-s) A u(s) d s
\end{aligned}
$$

is an isomorphism onto, where $D(A)$ is equipped with the graph norm. Indeed, since $A$ is closed, the space $H_{\text {per }}^{1, p}(0,2 \pi ; X) \cap L^{p}(0,2 \pi ; D(A))$ becomes a Banach space under the norm

$$
\|u\|:=\|u\|_{p}+\left\|u^{\prime}\right\|_{p}+\|A u\|_{p} .
$$

Appropriate analogues hold in the other cases. Such isomorphisms will be crucial in Section 4 for the treatment of the nonlinear equation. 
In the case of a Hilbert space, Theorem 2.9 takes a particularly simple form. It is remarkable that it corresponds essentially to the case where $X=\mathbb{C}$. Recall that under assumption (H0a) on $a(\cdot)$ and $b(\cdot)$, the sequences $\left(a_{k}\right)$ and $\left(b_{k}\right)$ are bounded.

Corollary 2.11. Let $H$ be a Hilbert space and $A: D(A) \subset H \rightarrow H$ be a closed linear operator. Assume that the function $a(\cdot) \in L^{1}\left(\mathbb{R}_{+}\right)$is such that $\left(\left(c_{0}-a_{k}\right)^{-1}\right)$ is bounded. Moreover, let $b(\cdot) \in L^{1}\left(\mathbb{R}_{+}\right)$. Then for $\mathcal{Y}=$ $L^{p}(0,2 \pi ; H), 1<p<\infty$, the following assertions are equivalent:

(i) For every $f \in L_{\text {per }}^{p}(\mathbb{R} ; H)$ there exists a unique strong $L^{p}$-solution of (2.1).

(ii) $\left\{d_{k}\right\}_{k \in \mathbb{Z}} \subset \varrho(A)$ and

$$
\sup _{k}\left\|\frac{i k}{c_{0}-a_{k}}\left(d_{k} I-A\right)^{-1}\right\|<\infty .
$$

This is a consequence of the validity of Plancherel's theorem.

3. Maximal regularity on periodic Lebesgue and Besov spaces. In this section, we give conditions that enable us to apply Theorem 2.9 in various situations by use of the operator-valued multiplier theorems established in [5], [3], [6]. Versions of the multiplier theorems on the real line can be found in [2], [18], [19] (this reference contains criteria implying the $R$-boundedness property for operator families), [31] and [32].

For $j \in \mathbb{N}$, denote by $r_{j}$ the $j$ th Rademacher function on $[0,1]$, i.e. $r_{j}(t)=\operatorname{sgn}\left(\sin \left(2^{j} \pi t\right)\right)$. For $x \in X$ we denote by $r_{j} \otimes x$ the vector-valued function $t \mapsto r_{j}(t) x$.

Definition 3.1. A family $\mathbf{T} \subset \mathcal{L}(X, Y)$ is called $R$-bounded if there exists $c_{q} \geq 0$ such that

$$
\left\|\sum_{j=1}^{n} r_{j} \otimes T_{j} x_{j}\right\|_{L^{q}(0,1 ; X)} \leq c_{q}\left\|\sum_{j=1}^{n} r_{j} \otimes x_{j}\right\|_{L^{q}(0,1 ; X)}
$$

for all $T_{1}, \ldots, T_{n} \in \mathbf{T}, x_{1}, \ldots, x_{n} \in X$ and $n \in \mathbb{N}$, where $1 \leq q<\infty$. We denote by $R_{q}(\mathbf{T})$ the smallest constant $c_{q}$ such that (3.1) holds.

Remark 3.2. (a) Let $\mathbf{S}, \mathbf{T} \subset \mathcal{L}(X)$ be $R$-bounded sets. Then $\mathbf{S} \cdot \mathbf{T}:=$ $\{S \cdot T: S \in \mathbf{S}, T \in \mathbf{T}\}$ is $R$-bounded and

$$
R_{p}(\mathbf{S} \cdot \mathbf{T}) \leq R_{p}(\mathbf{S}) \cdot R_{p}(\mathbf{T}) .
$$

(b) Also, each subset $M \subset \mathcal{L}(X)$ of the form $M=\{\lambda I: \lambda \in \Omega\}$ is $R$ bounded whenever $\Omega \subset \mathbb{C}$ is bounded. This follows from Kahane's contraction principle (see [5, Lemma 1.7]). We shall use this remark frequently. 
Remark 3.3. If $X=Y$ is a UMD space and $M_{k}=m_{k} I$ with $m_{k} \in \mathbb{C}$, then the condition

$$
\sup _{k}\left|m_{k}\right|+\sup _{k}\left|k\left(m_{k+1}-m_{k}\right)\right|<\infty
$$

implies that the set $\left\{M_{k}\right\}_{k \in \mathbb{Z}}$ is an $L^{p}$-multiplier (see [5] or [1, Theorem 4.4.3]). This is the vector-valued multiplier theorem (see e.g. [2]). In the context of Cauchy problems where one is dealing with the resolvent of a closed operator $A$, this would apply to the situation where $A$ is a multiplication operator on $L^{r}(\Omega, \mu)$ for $\Omega$ a measure space and $1<r<\infty$.

Another important notion is that of Fourier type for a Banach space. The Hausdorff-Young inequality states that for $1 \leq p \leq 2$, the Fourier transform maps $L^{p}(\mathbb{R}):=L^{p}(\mathbb{R} ; \mathbb{C})$ into $L^{p^{\prime}}(\mathbb{R})$ where $1 / p+1 / p^{\prime}=1$, with the convention that $p^{\prime}=\infty$ when $p=1$. In particular, when $p=2$, Plancherel's theorem holds. When $X$ is a Banach space and one considers $L^{p}(\mathbb{R} ; X)$, the situation is no longer the same. It is known that Plancherel's theorem holds if and only if $X$ is a Hilbert space (see [22]). For every Banach space, the Hausdorff-Young theorem holds with $p=1$. A Banach space is said to have nontrivial Fourier type if the Hausdorff-Young theorem holds true for some $p \in(1,2]$. By a result of Bourgain, UMD spaces are examples of spaces with nontrivial Fourier type (see [19], [3]). More generally, $B$-convex spaces, in particular superreflexive Banach spaces, have nontrivial Fourier type ([8, Proposition 3]). However, there exist nonreflexive Banach spaces with nontrivial Fourier type. Note that by a theorem of Pisier, a Banach space has nontrivial Fourier type if and only if it has nontrivial Rademacher type. The notion of Rademacher type plays an important role in [5].

We introduce two other conditions which imply (H0) in various instances.

Let $\left(s_{k}\right)_{k \in \mathbb{Z}}$ be a sequence of complex numbers. We consider the following conditions:

(H1a) $\quad\left\{s_{k}\right\}_{k \in \mathbb{Z}},\left\{k\left(s_{k+1}-s_{k}\right)\right\}_{k \in \mathbb{Z}}$ are bounded sequences.

(H1b) $\quad\left\{\left(c_{0}-s_{k}\right)^{-1}\right\}_{k \in \mathbb{Z}}$ is a bounded sequence.

(H2) $\quad\left\{k s_{k}\right\}$ and $\left\{k^{2}\left(s_{k+1}-2 s_{k}+s_{k-1}\right)\right\}$ are bounded sequences.

When we refer simply to (H1), we mean (H1a)-(H1b).

We note that (H1) puts conditions on the first order differences whereas (H2) is concerned with second order differences.

Remark 3.4. We note that (H2) implies (H1a). Also, (H1a) implies 1regularity and (H2) implies 2-regularity in the sense of [20, Definition 2.6]. Conversely, whenever $\left\{s_{k}\right\}_{k \in \mathbb{Z}}$ corresponds to the Fourier transform of a given function $a \in L^{1}(\mathbb{R})$ we obtain, by the Riemann-Lebesgue lemma, that 1-regularity implies condition (H1a) and hence condition (H1) is in fact equivalent to 1-regularity (under the additional assumption that $\widetilde{a}(i k) \neq c_{0}$ 
for all $k \in \mathbb{Z}$ ). Analogously, if $\left\{s_{k}\right\}_{k \in \mathbb{Z}}$ corresponds to the Fourier transform of a given function $a \in \mathrm{BV}\left(\mathbb{R}_{+}\right)$such that $\dot{a} \in L^{1}(\mathbb{R})$ then (H2) is equivalent to 2-regularity.

On the other hand, condition (H2) is satisfied by the sequence $s_{k}=\widetilde{a}(i k)$ corresponding to the kernels $a(t)=\sum_{j=1}^{n} c_{j} e^{-\alpha_{j} t}$, where $\alpha_{j}, c_{j}$ are positive real numbers and $n \in \mathbb{N}$, which arise in applications to viscoelasticity.

REMARK 3.5. If $a \in W^{1,1}\left(\mathbb{R}_{+}\right)$is a positive function and

$$
c_{0}-\int_{0}^{\infty} a(s) d s>0
$$

then (H1) is satisfied with $s_{k}=\widetilde{a}(i k)$. In fact, first note that $a(\cdot)$ has a continuous representative which we continue to denote by $a(\cdot)$ (see $[9$, Théorème VIII.2]). This representative is continuous on $[0, \infty)$. More precisely it has a limit as $t \rightarrow 0^{+}$. Moreover, we have $a(t)-a(s)=\int_{s}^{t} a^{\prime}(\sigma) d \sigma, s, t \in[0, \infty)$. Since $a^{\prime} \in L^{1}\left(\mathbb{R}_{+}\right)$, the limit $a(\infty):=\lim _{t \rightarrow \infty} a(t)$ exists and $a(\infty)=0$. Using integration by parts we obtain

$$
k \widehat{a}(i k)=-a(0)-i \int_{0}^{\infty} e^{-i k t} a^{\prime}(t) d t .
$$

Hence

$k\left(s_{k+1}-s_{k}\right)=k \int_{0}^{\infty} e^{-i k t}\left(e^{-i t}-1\right) a(t) d t=-s_{k+1}-i \int_{0}^{\infty} e^{-i k t}\left(e^{-i t}-1\right) a^{\prime}(t) d t$.

Since $g(t):=\left(e^{-i t}-1\right) a^{\prime}(t)$ belongs again to $L^{1}\left(\mathbb{R}_{+}\right)$we see that the sequence $\left(k\left(s_{k+1}-s_{k}\right)\right)$ is bounded. On the other hand, by the Riemann-Lebesgue lemma, $\widetilde{a}(i k) \rightarrow 0$ and since $|\widetilde{a}(i k)| \leq \widetilde{a}(0)<c_{0}$ we get $\widehat{a}(i k) \neq c_{0}$ for all $k \in \mathbb{Z}$, and $\left(a_{k}\right)$ and $\left(\left(c_{0}-a_{k}\right)^{-1}\right)$ are also bounded sequences.

Note that the conditions established in the above remark are satisfied in practical problems (see e.g. [25] for a physical motivation of (3.2)) and by the following examples:

(a) $a(t)=t^{\delta} e^{-\alpha t}$ where $\alpha>0$ and $\delta \in[0, \infty)$.

(b) $a(t)=e^{-\alpha t} /(t+\beta)^{k}$ where $k \in \mathbb{N}, \beta>0$ and $\alpha>0$.

(c) $a(\cdot)$ is a completely monotone function. In this case, by Bernstein's theorem (see e.g. [4, Section 2.7]),

$$
a(t)=\int_{0}^{\infty} e^{-t x} \mu(d x)
$$

where $\mu$ is a suitable positive Borel measure. We make the assumption that

$$
\int_{0}^{\infty} \frac{\mu(d t)}{t}<c_{0}<\infty, \quad \int_{0}^{\infty} \mu(d t)<\infty .
$$


Note that these conditions imply that $a$ belongs to $W^{1,1}\left(\mathbb{R}_{+}\right)$. Examples of this type may be found in [23], [26].

We remark that the positivity of $a(\cdot)$ or $b(\cdot)$ in the above examples is by no means necessary. Since by the Riemann-Lebesgue lemma $\lim _{|k| \rightarrow \infty} a_{k}=0$ and $c_{0} \neq 0$, it suffices to enssure that $a_{k} \neq c_{0}, k \in \mathbb{Z}$. For example if $a(t)=e^{-\alpha t} \sin \omega t, t \geq 0$ (where $\left.\alpha>0\right)$, then

$$
a_{k}=\frac{\omega}{(i k+\alpha)^{2}+\omega^{2}}, \quad k \in \mathbb{Z} .
$$

In this case, it is easy to see that $a_{k}-c_{0} \neq 0, k \in \mathbb{Z}$, provided $\left(\alpha^{2}+\omega^{2}\right) c_{0} \neq \omega$.

Now, suppose $a \in W^{2,1}\left(\mathbb{R}_{+}\right)$. Then, from the identity

$$
s_{k+1}-2 s_{k}+s_{k-1}=2 \int_{0}^{\infty} e^{-i k t}(\cos t-1) a(t) d t
$$

and integrating by parts twice, we deduce that (H2) is satisfied.

In order to relate conditions (H1) and (H2) to the assumption (H0) of Section 2, we have the following lemma.

Lemma 3.6. (1) Let $X$ be a UMD space. Assume that $\left(a_{k}\right)$ satisfies assumption ( $\mathrm{H} 1)$ and $\left(b_{k}\right)$ satisfies $(\mathrm{H} 1 \mathrm{a})$. Then the sequences $\left\{\left(c_{0}-a_{k}\right) I\right\}_{k \in \mathbb{Z}},\left\{\left(\left(c_{0}-a_{k}\right)^{-1}\right) I\right\}_{k \in \mathbb{Z}}$ and $\left\{\left(1+b_{k}\right) I\right\}_{k \in \mathbb{Z}}$ are $L^{p_{-}}$ multipliers as well as $B_{p, q}^{s}$-multipliers for $1<p, q<\infty$ and $s \in \mathbb{R}$.

(2) If $X$ is an arbitrary Banach space and $\left(a_{k}\right),\left(b_{k}\right)$ satisfy assumption $(\mathrm{H} 2)$ and $\left(a_{k}\right)$ satisfies $(\mathrm{H} 1 \mathrm{~b})$, then the sequences $\left\{\left(c_{0}-a_{k}\right) I\right\}_{k \in \mathbb{Z}}$, $\left\{\left(\left(c_{0}-a_{k}\right)^{-1}\right) I\right\}_{k \in \mathbb{Z}}$ and $\left\{\left(1+b_{k}\right) I\right\}_{k \in \mathbb{Z}}$ are $B_{p, q}^{s}$-multipliers for $1 \leq$ $p, q \leq \infty$ and $-\infty<s<\infty$. In particular they are $\mathcal{C}^{s}$-multipliers.

Note that the conclusion in (1) about $B_{p, q}^{s}$-multipliers is valid more generally whenever $X$ has nontrivial Fourier type (see [18] and [3]).

This follows from [20, Lemmas 2.9 and 3.8].

Remark 3.7. Note that for $s>0, B_{\infty, \infty}^{s}=\mathcal{C}^{s}$. The latter are the Hölder-Zygmund spaces. They coincide with the familiar Hölder spaces $C^{s}$ when $s \notin \mathbb{N}$. More specifically, for $0<s<1$, an equivalent norm on $B_{\infty, \infty}^{s}$ is given by

$$
\|f\|_{s}=\sup \left\{\frac{\left\|f(t)-f\left(t^{\prime}\right)\right\|}{\left|t-t^{\prime}\right|^{s}}: t, t^{\prime} \in[0,2 \pi], t \neq t^{\prime}\right\}+\|f\|_{\infty}
$$

corresponding to the familiar Hölder space of exponent $s$. On the other hand, when $s=1$, an equivalent norm on $B_{\infty, \infty}^{1}$ is given by

$$
\|f\|_{s}=\sup _{t \in[0,2 \pi]} \sup _{h \neq 0}\left\{\frac{\|f(t+h)-2 f(t)+f(t-h)\|}{|h|}\right\}+\|f\|_{\infty} .
$$


For more on this and more generally the description of the Besov spaces using finite differences, we refer to [6, Section 2]. The monograph [30] contains various other characterizations (notably those using semigroup theory and interpolation, the modulus of continuity, and approximation theory) and extends the definition to the range $0<p, q \leq \infty, s \in \mathbb{R}$.

We give the definition of well-posedness for the integro-differential equation (2.1).

Definition 3.8. We shall say that (2.1) is well posed in $\mathcal{Y}$ if for every $f \in \mathcal{Y}$, there exists a unique strong $\mathcal{Y}$-solution of (2.1).

Depending on the context, it will be clear which Banach space $X$ and which scale $\left(L^{p}\right.$ or $\left.B_{p, q}^{s}\right)$ is concerned.

We begin with the following result for UMD spaces. Recall from Section 2 that

$$
M_{k}:=\frac{i k}{c_{0}-a_{k}}\left(d_{k} I-A\right)^{-1}, \quad d_{k}=\frac{i k\left(\gamma_{0}+b_{k}\right)+\gamma_{\infty}}{c_{0}-a_{k}} .
$$

Theorem 3.9. Let $X$ be a UMD space. Assume that $a(\cdot), b(\cdot) \in L^{1}\left(\mathbb{R}_{+}\right)$ and the sequences $\left(a_{k}\right)$ and $\left(b_{k}\right)$ satisfy $(\mathrm{H} 1)$ and $(\mathrm{H} 1 \mathrm{a})$ respectively. Then the following statements are equivalent:

(i) $\left\{d_{k}\right\}_{k \in \mathbb{Z}} \subseteq \varrho(A)$ and $\left(M_{k}\right)$ is $R$-bounded.

(ii) Problem (2.1) is well posed in $L^{p}(0,2 \pi ; X)$ for all $1<p<\infty$.

Proof. (ii) $\Rightarrow($ i). Assume that (2.1) is well posed. Then by Theorem 2.9, $\left\{b_{k}\right\}_{k \in \mathbb{Z}} \subseteq \varrho(A)$ and $\left(M_{k}\right)$ is an $L^{p}$-multiplier. The $R$-boundedness of $\left(M_{k}\right)$ now follows from [5, Proposition 1.11].

(i) $\Rightarrow$ (ii). In view of Theorem 2.9 , it suffices to show that $\left(M_{k}\right)$ is an $L^{p_{-}}$ multiplier. Now according to the operator-valued Marcinkiewicz multiplier theorem ([5, Theorem 1.3]), it is enough to prove that the operator-valued sequences $\left(M_{k}\right)$ and $k\left(M_{k+1}-M_{k}\right)$ are $R$-bounded. Furthermore, since $M_{k}=$ $i k\left(c_{0}-a_{k}\right)^{-1}\left(d_{k} I-A\right)^{-1}$ and by our assumption that $\left(\left(c_{0}-a_{k}\right)^{-1} I\right)$ is a multiplier, by Remark 2.3 it suffices to show that

$$
\mu_{k}:=i k\left(d_{k}-A\right)^{-1}
$$

is an $L^{p}$-multiplier. So we check that $\left(\mu_{k}\right)$ and $k\left(\mu_{k+1}-\mu_{k}\right)$ are $R$-bounded. The $R$-boundedness of $\left(\mu_{k}\right)$ follows from that of $\left(M_{k}\right)$ by Remark 3.2. As for $k\left(\mu_{k+1}-\mu_{k}\right)$, we have, using the resolvent equation,

$$
\begin{aligned}
k\left(\mu_{k+1}-\mu_{k}\right) & =k\left[i(k+1)\left(d_{k+1}-A\right)^{-1}-i k\left(d_{k}-A\right)^{-1}\right] \\
& =i k\left[(k+1)\left(\left(d_{k+1}-A\right)^{-1}-\left(d_{k}-A\right)^{-1}\right)+\left(d_{k}-A\right)^{-1}\right] \\
& =i k(k+1)\left(\left(d_{k+1}-A\right)^{-1}-\left(d_{k}-A\right)^{-1}\right)+i k\left(d_{k}-A\right)^{-1} \\
& =-i k(k+1)\left(d_{k+1}-d_{k}\right)\left(d_{k+1}-A\right)^{-1}\left(d_{k}-A\right)^{-1}+i k\left(d_{k}-A\right)^{-1} \\
& =i\left(d_{k+1}-d_{k}\right) \mu_{k} \mu_{k+1}+\mu_{k} .
\end{aligned}
$$


By Kahane's contraction principle [5, Lemma 1.7], it suffices to show that the sequence $\left(d_{k+1}-d_{k}\right)$ is bounded. But we have

$$
\begin{aligned}
d_{k}-d_{k+1} & =\frac{i k\left(\gamma_{0}+b_{k}\right)+\gamma_{\infty}}{c_{0}-a_{k}}-\frac{i(k+1)\left(\gamma_{0}+b_{k+1}\right)+\gamma_{\infty}}{c_{0}-a_{k+1}} \\
& =\gamma_{\infty}\left(\frac{1}{c_{0}-a_{k}}-\frac{1}{c_{0}-a_{k+1}}\right)+\frac{i k\left(\gamma_{0}+b_{k}\right)}{c_{0}-a_{k}}-\frac{i(k+1)\left(\gamma_{0}+b_{k+1}\right)}{c_{0}-a_{k+1}} .
\end{aligned}
$$

We need only look at the last two terms. We have

$$
\frac{k\left(\gamma_{0}+b_{k}\right)}{c_{0}-a_{k}}-\frac{(k+1)\left(\gamma_{0}+b_{k+1}\right)}{c_{0}-a_{k+1}}=k\left[\frac{\gamma_{0}+b_{k}}{c_{0}-a_{k}}-\frac{\gamma_{0}+b_{k+1}}{c_{0}-a_{k+1}}\right]-\frac{\gamma_{0}+b_{k+1}}{c_{0}-a_{k+1}} .
$$

Since by assumption, the two sequences $\left(\left(c_{0}-a_{k}\right)^{-1}\right)$ and $\left(b_{k}\right)$ are bounded, we need only examine

$$
\begin{aligned}
k\left[\frac{\gamma_{0}+b_{k}}{c_{0}-a_{k}}-\frac{\gamma_{0}+b_{k+1}}{c_{0}-a_{k+1}}\right]= & \frac{-k\left[b_{k} a_{k+1}-b_{k+1} a_{k}\right]}{\left(c_{0}-a_{k}\right)\left(c_{0}-a_{k+1}\right)}-\frac{\gamma_{0} k\left(a_{k+1}-a_{k}\right)}{\left(c_{0}-a_{k}\right)\left(c_{0}-a_{k+1}\right)} \\
& +\frac{c_{0} k\left(b_{k}-b_{k+1}\right)}{\left(c_{0}-a_{k}\right)\left(c_{0}-a_{k+1}\right)} \\
= & \frac{-k\left[\left(b_{k}-b_{k+1}\right) a_{k+1}+b_{k+1}\left(a_{k+1}-a_{k}\right)\right]}{\left(c_{0}-a_{k}\right)\left(c_{0}-a_{k+1}\right)} \\
& +\frac{-\gamma_{0} k\left(a_{k+1}-a_{k}\right)+c_{0} k\left(b_{k}-b_{k+1}\right)}{\left(c_{0}-a_{k}\right)\left(c_{0}-a_{k+1}\right)} \\
= & \frac{-k\left(b_{k}-b_{k+1}\right) a_{k+1}}{\left(c_{0}-a_{k}\right)\left(c_{0}-a_{k+1}\right)}-\frac{b_{k+1} k\left(a_{k+1}-a_{k}\right)}{\left(c_{0}-a_{k}\right)\left(c_{0}-a_{k+1}\right)} \\
& -\frac{\gamma_{0} k\left(a_{k+1}-a_{k}\right)+c_{0} k\left(b_{k}-b_{k+1}\right)}{\left(c_{0}-a_{k}\right)\left(c_{0}-a_{k+1}\right)} .
\end{aligned}
$$

In view of assumption (H1), the claim is established.

From this theorem, we deduce the following result for $B_{p, q}^{s}$-solutions.

Theorem 3.10. Let $X$ be a Banach space with nontrivial Fourier type. Assume that $a(\cdot), b(\cdot) \in L^{1}\left(\mathbb{R}_{+}\right)$and the sequences $\left(a_{k}\right)$ and $\left(b_{k}\right)$ satisfy $(\mathrm{H} 1)$ and (H1a) respectively. Then for $s \in \mathbb{R}$ and $1 \leq p, q \leq \infty$, the following are equivalent:

(i) $\left\{d_{k}\right\}_{k \in \mathbb{Z}} \subseteq \varrho(A)$ and $\left(M_{k}\right)$ is bounded.

(ii) Problem (2.1) is well posed in $B_{p, q}^{s}(0,2 \pi ; X)$.

Proof. The proof follows the same lines as that of the preceding theorem and uses [3, Theorem 3.4 and Remark 3.6] and [6, Theorem 3.5] (see also [18]). For the boundedness of $\left(M_{k}\right)$, one uses [20, proof of Proposition 3.4] or $[6]$. 
Remark 3.11. Fejér's theorem (see [5, Proposition 1.1] or [4, Theorem 4.2.19]) allows us to construct the solution $u(\cdot)$ given by the above theorems. In fact, in the case where $\mathcal{Y}=L^{p}(0,2 \pi ; X)$ we have

$$
u(\cdot)=\lim _{n \rightarrow \infty} \frac{1}{n+1} \sum_{m=0}^{n} \sum_{k=-m}^{m} e_{k}(\cdot) R\left(d_{k}, A\right)\left(c_{0}-a_{k}\right)^{-1} \widehat{f}(k)
$$

with convergence in $L^{p}(0,2 \pi ; X)$.

We now consider the problem of well-posedness in Besov spaces for arbitrary Banach spaces. For this, assumption (H1) is no longer sufficient. It is proved in [6, Theorem 3.2] that if for any sequence $\left(M_{k}\right)_{k \in \mathbb{Z}} \subset \mathcal{L}(X)$, the so-called variational Marcinkiewicz condition, that is,

$$
\sup _{k \in \mathbb{Z}}\left\|M_{k}\right\|+\sup _{j \geq 0}\left(\sum_{2^{j} \leq|k|<2^{j+1}}\left\|M_{k+1}-M_{k}\right\|\right)<\infty,
$$

implies that $\left(M_{k}\right)$ is a $B_{p, q}^{s}$-multiplier, then $1<p<\infty$ and $X$ is a UMD space. The converse is also true.

For Banach spaces with nontrivial Fourier type, a condition which implies that $\left(M_{k}\right)$ is a Fourier multiplier for the scale $B_{p, q}^{s}, s \in \mathbb{R}, 1 \leq p, q \leq \infty$, is the Marcinkiewicz condition of order one:

$$
\sup _{k \in \mathbb{Z}}\left(\left\|M_{k}\right\|+\left\|k\left(M_{k+1}-M_{k}\right)\right\|\right)<\infty,
$$

which is used in the proof of Theorem 3.10.

For arbitrary Banach spaces, a Marcinkiewicz condition of order two is needed, namely,

$$
\sup _{k \in \mathbb{Z}}\left(\left\|M_{k}\right\|+\left\|k\left(M_{k+1}-M_{k}\right)\right\|+k^{2}\left\|M_{k+1}-2 M_{k}+M_{k-1}\right\|\right)<\infty .
$$

Our next result uses this condition to obtain maximal regularity of problem (2.1) in general Banach spaces. Here, condition (H2) is needed and the result is established for the Besov scale $B_{p, q}^{s}, s \in \mathbb{R}, 1 \leq p, q \leq \infty$.

Theorem 3.12. Let $X$ be a Banach space. Assume that $a(\cdot), b(\cdot) \in$ $L^{1}\left(\mathbb{R}_{+}\right)$, the sequences $\left(a_{k}\right)$ and $\left(b_{k}\right)$ satisfy $(\mathrm{H} 2)$, and $\left(a_{k}\right)$ satisfies $(\mathrm{H} 1 \mathrm{~b})$. Then for $s>0$ and $1 \leq p, q \leq \infty$, the following are equivalent:

(i) $\left\{d_{k}\right\}_{k \in \mathbb{Z}} \subseteq \varrho(A)$ and $\left(M_{k}\right)$ is bounded.

(ii) Problem (2.1) is well posed in $B_{p, q}^{s}(0,2 \pi ; X)$.

Proof. (ii) $\Rightarrow$ (i) follows from e.g. [3, Lemma 3.2] or one can argue as in the proof of [6, Theorem 3.2]. As above, to show that $\left(M_{k}\right)$ is bounded, we use [20, proof of Proposition 3.4] or [6].

(i) $\Rightarrow$ (ii). As already noted, (H2) implies (H1a). Hence the verification of the Marcinkiewicz condition of order one is similar to what was done in 
the proof of Theorem 3.9, and is in fact easier, owing to the boundedness of $\left(k a_{k}\right)$ and $\left(k b_{k}\right)$. It remains to prove that

$$
\sup _{k \in \mathbb{Z}}\left(k^{2}\left\|M_{k+1}-2 M_{k}+M_{k-1}\right\|\right)<\infty .
$$

By Lemma 3.6, (H2) implies that $\left(a_{k} I\right)$ and $\left(b_{k} I\right)$ are both $B_{p, q}^{s}$-multipliers. As in the proof of Theorem 3.9, we again set $\mu_{k}=i k\left(d_{k}-A\right)^{-1}$. We have to verify $(3.12)$ on $\left(\mu_{k}\right)$. Note in particular that $\left(\mu_{k}\right)$ is bounded. For $k \in \mathbb{Z}$, we have, setting $G_{k}:=k^{2}\left(\mu_{k+1}-2 \mu_{k}+\mu_{k-1}\right)$,

$$
\begin{aligned}
G_{k}= & i k^{2}\left[(k+1)\left(d_{k+1}-A\right)^{-1}-2 k\left(d_{k}-A\right)^{-1}+(k-1)\left(d_{k-1}-A\right)^{-1}\right] \\
= & i k^{2}\left\{\left[k\left(\left(d_{k+1}-A\right)^{-1}-\left(d_{k}-A\right)^{-1}\right)\right]\right. \\
& \left.-\left[k\left(\left(d_{k}-A\right)^{-1}-\left(d_{k-1}-A\right)^{-1}\right)\right]+\left[\left(d_{k+1}-A\right)^{-1}-\left(d_{k-1}-A\right)^{-1}\right]\right\} \\
= & i k^{2}\left[k\left(\left(d_{k+1}-A\right)^{-1}-\left(d_{k}-A\right)^{-1}\right)\right]-i k^{2}\left[k\left(\left(d_{k}-A\right)^{-1}-\left(d_{k-1}-A\right)^{-1}\right)\right] \\
& +i k^{2}\left[\left(d_{k+1}-A\right)^{-1}-\left(d_{k-1}-A\right)^{-1}\right] \\
= & : G_{k}^{1}-G_{k}^{2}+G_{k}^{3} .
\end{aligned}
$$

We first examine the last square brackets. Using the resolvent equation, we obtain, with $G_{k}^{3}:=i k^{2}\left[\left(d_{k+1}-A\right)^{-1}-\left(d_{k-1}-A\right)^{-1}\right]$,

$$
\begin{aligned}
G_{k}^{3}= & i k^{2}\left(d_{k-1}-d_{k+1}\right)\left(d_{k+1}-A\right)^{-1}\left(d_{k-1}-A\right)^{-1} \\
= & i\left(d_{k-1}-d_{k+1}\right)(k+1)\left(d_{k+1}-A\right)^{-1}(k-1)\left(d_{k-1}-A\right)^{-1} \\
& \quad+i\left(d_{k-1}-d_{k+1}\right)\left(d_{k+1}-A\right)^{-1}\left(d_{k-1}-A\right)^{-1} \\
= & -i\left(d_{k-1}-d_{k+1}\right) \mu_{k+1} \mu_{k-1}+i\left(d_{k-1}-d_{k+1}\right) \nu_{k-1} \nu_{k+1}
\end{aligned}
$$

where we have set

$$
\nu_{k}=\left(d_{k}-A\right)^{-1}, \quad k \in \mathbb{Z} .
$$

Since the sequence $\left(\mu_{k}\right)$ is bounded and $\mu_{k}=i k \nu_{k}, k \in \mathbb{Z}$, it follows that $\left(\nu_{k}\right)$ is bounded as well. It remains to show that $\left(d_{k+1}-d_{k-1}\right)$ is bounded. But this follows from the boundedness of the sequence $\left(d_{k}-d_{k+1}\right)$ established in the course of the proof of Theorem 3.9, and the relation $d_{k+1}-d_{k-1}=$ $\left(d_{k+1}-d_{k}\right)+\left(d_{k}-d_{k-1}\right)$.

It remains to estimate $G_{k}^{1}-G_{k}^{2}$. We shall first express this using the first order differences $d_{k+1}-d_{k}$, the second order differences $d_{k+1}-2 d_{k}+d_{k-1}$ and the sequences $\left(\mu_{k}\right)_{k \in \mathbb{Z}},\left(\nu_{k}\right)_{k \in \mathbb{Z}}$ defined above. Making use once more of the resolvent equation yields

$$
\begin{aligned}
-i\left(G_{k}^{1}-\right. & \left.G_{k}^{2}\right) \\
= & k^{3}\left[\left(d_{k+1}-A\right)^{-1}-2\left(d_{k}-A\right)^{-1}+\left(d_{k-1}-A\right)^{-1}\right] \\
= & k^{3}\left[\left(d_{k+1}-A\right)^{-1}-\left(d_{k}-A\right)^{-1}+\left(d_{k-1}-A\right)^{-1}-\left(d_{k}-A\right)^{-1}\right] \\
= & k^{3}\left(d_{k}-d_{k+1}\right)\left(d_{k}-A\right)^{-1}\left(d_{k+1}-A\right)^{-1} \\
& \quad-k^{3}\left(d_{k-1}-d_{k}\right)\left(d_{k}-A\right)^{-1}\left(d_{k-1}-A\right)^{-1}
\end{aligned}
$$




$$
\begin{aligned}
= & k\left(d_{k}-A\right)^{-1} k^{2}\left[\left(d_{k}-d_{k+1}\right)\left(d_{k+1}-A\right)^{-1}-\left(d_{k}-d_{k-1}\right)\left(d_{k-1}-A\right)^{-1}\right] \\
= & k\left(d_{k}-A\right)^{-1} k^{2}\left[\left(d_{k}-d_{k+1}\right)\left(\left(d_{k+1}-A\right)^{-1}-\left(d_{k-1}-A\right)^{-1}\right)\right. \\
& \left.+\left(d_{k+1}-2 d_{k}+d_{k-1}\right)\left(d_{k-1}-A\right)^{-1}\right] \\
= & k\left(d_{k}-A\right)^{-1}\left[k^{2}\left(d_{k}-d_{k+1}\right)\left(d_{k-1}-d_{k+1}\right)\left(d_{k+1}-A\right)^{-1}\left(d_{k-1}-A\right)^{-1}\right) \\
& \left.+k\left(d_{k+1}-2 d_{k}+d_{k-1}\right) k\left(d_{k-1}-A\right)^{-1}\right] \\
= & -i \mu_{k}\left[\left(d_{k}-d_{k+1}\right)\left(d_{k-1}-d_{k+1}\right)\left(\mu_{k-1} \mu_{k+1}+\nu_{k-1} \nu_{k+1}\right)\right. \\
& \left.-i k\left(d_{k+1}-2 d_{k}+d_{k-1}\right)\left(\mu_{k-1}+\nu_{k-1}\right)\right] .
\end{aligned}
$$

In view of the boundedness of $\mu_{k}:=i k\left(d_{k}-A\right)^{-1}, \nu_{k}:=\left(d_{k}-A\right)^{-1}$ it remains to verify that the sequence $\left(k\left(d_{k+1}-2 d_{k}+d_{k-1}\right)\right), k \in \mathbb{Z}$, is bounded. This will follow from (H2). In fact,

$$
\begin{aligned}
k & \left(d_{k+1}-2 d_{k}+d_{k-1}\right) \\
= & k\left[\frac{i(k+1)\left(\gamma_{0}+b_{k+1}\right)+\gamma_{\infty}}{c_{0}-a_{k+1}}-2 \frac{i k\left(\gamma_{0}+b_{k}\right)+\gamma_{\infty}}{c_{0}-a_{k}}+\frac{i(k-1)\left(\gamma_{0}+b_{k-1}\right)+\gamma_{\infty}}{c_{0}-a_{k-1}}\right] \\
= & k\left[\frac{i(k+1)\left(\gamma_{0}+b_{k+1}\right)}{c_{0}-a_{k+1}}-2 \frac{i k\left(\gamma_{0}+b_{k}\right)}{c_{0}-a_{k}}+\frac{i(k-1)\left(\gamma_{0}+b_{k-1}\right)}{c_{0}-a_{k-1}}\right] \\
& +k\left[\frac{\gamma_{\infty}}{c_{0}-a_{k+1}}-2 \frac{\gamma_{\infty}}{c_{0}-a_{k}}+\frac{\gamma_{\infty}}{c_{0}-a_{k-1}}\right] \\
= & i k^{2}\left[\frac{\gamma_{0}+b_{k+1}}{c_{0}-a_{k+1}}-2 \frac{\gamma_{0}+b_{k}}{c_{0}-a_{k}}+\frac{\gamma_{0}+b_{k-1}}{c_{0}-a_{k-1}}\right]+i k\left[\frac{\gamma_{0}+b_{k+1}}{c_{0}-a_{k+1}}-\frac{\gamma_{0}+b_{k-1}}{c_{0}-a_{k-1}}\right] \\
& +k\left[\frac{\gamma_{\infty}}{c_{0}-a_{k+1}}-2 \frac{\gamma_{\infty}}{c_{0}-a_{k}}+\frac{\gamma_{\infty}}{c_{0}-a_{k-1}}\right] \\
= & i k^{2}\left[\frac{\gamma_{0}}{c_{0}-a_{k+1}}-2 \frac{\gamma_{0}}{c_{0}-a_{k}}+\frac{\gamma_{0}}{c_{0}-a_{k-1}}\right] \\
& +i k^{2}\left[\frac{b_{k+1}}{c_{0}-a_{k+1}}-2 \frac{b_{k}}{c_{0}-a_{k}}+\frac{b_{k-1}}{c_{0}-a_{k-1}}\right] \\
& +i k\left[\frac{\gamma_{0}+b_{k+1}}{c_{0}-a_{k+1}}-\frac{\gamma_{0}+b_{k-1}}{c_{0}-a_{k-1}}\right]+k\left[\frac{\gamma_{\infty}}{c_{0}-a_{k+1}}-2 \frac{\gamma_{\infty}}{c_{0}-a_{k}}+\frac{\gamma_{\infty}}{c_{0}-a_{k-1}}\right] .
\end{aligned}
$$

For the verification concerning the first and last brackets, we refer to the proof of [20, Lemma 3.8]. The third bracket is handled in a similar way to our verification of the boundedness of $\left(d_{k+1}-d_{k}\right)$. In fact, 


$$
\begin{gathered}
\frac{i k\left(\gamma_{0}+b_{k+1}\right)}{c_{0}-a_{k+1}}-\frac{i k\left(\gamma_{0}+b_{k-1}\right)}{c_{0}-a_{k-1}} \\
=i k \frac{\gamma_{0}\left(a_{k-1}-a_{k+1}\right)+c_{0}\left(b_{k+1}-b_{k-1}\right)+a_{k+1} b_{k-1}-a_{k-1} b_{k+1}}{\left(c_{0}-a_{k-1}\right)\left(c_{0}-a_{k+1}\right)} \\
=\frac{\gamma_{0} i k\left(a_{k-1}-a_{k+1}\right)+i k c_{0}\left(b_{k+1}-b_{k-1}\right)}{\left(c_{0}-a_{k-1}\right)\left(c_{0}-a_{k+1}\right)} \\
\quad+\frac{i k\left(a_{k+1}-a_{k-1}\right) b_{k+1}+a_{k+1}\left(b_{k-1}-b_{k+1}\right)}{\left(c_{0}-a_{k-1}\right)\left(c_{0}-a_{k+1}\right)} .
\end{gathered}
$$

We now consider the remaining bracket:

$$
k^{2}\left[\frac{b_{k+1}}{c_{0}-a_{k+1}}-2 \frac{b_{k}}{c_{0}-a_{k}}+\frac{b_{k-1}}{c_{0}-a_{k-1}}\right]=\frac{U_{k}}{\left(c_{0}-a_{k-1}\right)\left(c_{0}-a_{k}\right)\left(c_{0}-a_{k+1}\right)},
$$

where

$$
\begin{aligned}
U_{k}= & k^{2}\left[b_{k+1}\left(c_{0}^{2}-c_{0}\left(a_{k}+a_{k-1}\right)+a_{k} a_{k-1}\right)\right. \\
& -2 b_{k}\left(c_{0}^{2}-c_{0}\left(a_{k+1}+a_{k-1}\right)+a_{k+1} a_{k-1}\right) \\
& \left.+b_{k-1}\left(c_{0}^{2}-c_{0}\left(a_{k}+a_{k+1}\right)+a_{k} a_{k+1}\right)\right] \\
= & k^{2} c_{0}^{2}\left(b_{k+1}-2 b_{k}+b_{k-1}\right)-k^{2}\left[b_{k+1}\left(c_{0}\left(a_{k}+a_{k-1}\right)-a_{k} a_{k-1}\right)\right. \\
& \left.+2 b_{k}\left(c_{0}\left(a_{k+1}+a_{k-1}\right)-a_{k+1} a_{k-1}\right)-b_{k-1}\left(c_{0}\left(a_{k}+a_{k+1}\right)-a_{k} a_{k+1}\right)\right] .
\end{aligned}
$$

The sequence $k^{2}\left(b_{k+1}-2 b_{k}+b_{k+1}\right)$ is bounded since $\left(b_{k}\right)$ satisfies assumption (H2). For the remaining terms, we use the boundedness of $\left(k a_{k}\right)$ and $\left(k b_{k}\right)$ from assumption (H2). The proof of the theorem is complete.

A key feature in the results presented in this section is that they only involve boundedness of the resolvent of the operator $A$ (except in the $L^{p}$ case where $R$-boundedness is needed). This is of course due to the many implications of the resolvent equation. Another instance where the special nature of the resolvent plays a key role is the inversion of the vector-valued Laplace transform and its consequences for semigroup theory as developed in [4].

ExAmple 3.13. Define

$$
\begin{aligned}
\varrho_{a, b}(A):=\left\{\lambda \in \mathbb{C}: \frac{\lambda}{c_{0}-\widetilde{a}(\lambda)}\left(\frac{\lambda\left(\gamma_{0}+\widetilde{b}(\lambda)\right)+\gamma_{\infty}}{c_{0}-\widetilde{a}(\lambda)}-A\right)^{-1}\right. & \text { exists } \\
& \text { in } \mathcal{L}(X, D(A))\}
\end{aligned}
$$

(where $D(A)$ is equipped with the graph norm) and assume that the nonresonance condition (compare [15, Section 4] where the Laplace transform of $g$ is denoted by $\widehat{g}$ )

$$
i \mathbb{Z} \subseteq \varrho_{a, b}(A)
$$

is satisfied. Then $\left\{d_{k}\right\}_{k \in \mathbb{Z}} \subseteq \varrho(A)$. 
Suppose $\gamma_{0} \neq 0$ and the pair $(A, d)$ admits a bounded analytic resolvent (see [26, Chapter I, Section 2]), with $d$ the unique solution to the scalar convolution equation

$$
\gamma_{0} d(t)+\int_{0}^{t} b(t-s) d(s) d s+\gamma_{\infty} \int_{0}^{t} d(s) d s=c_{0}-\int_{0}^{t} a(s) d s .
$$

Since

$$
\frac{i k}{i k\left(\gamma_{0}+b_{k}\right)+\gamma_{\infty}} \rightarrow \frac{1}{\gamma_{0}} \quad \text { as } k \rightarrow \infty
$$

we obtain

$$
\sup _{k}\left\|M_{k}\right\|=\sup _{k}\left\|\frac{i k}{i k\left(\gamma_{0}+b_{k}\right)+\gamma_{\infty}} d_{k}\left(d_{k}-A\right)^{-1}\right\|<\infty .
$$

EXAMPLE 3.14. Let $A$ be a closed linear operator on $X$. Assume that the resolvent set $\varrho(A)$ of $A$ contains the sector $\Sigma=\{\lambda \in \mathbb{C}: \lambda \neq 0,|\arg (\lambda)|<\theta\}$ with $\theta \in(\pi / 2, \pi)$ and there exists $M>0$ such that

$$
\left\|\lambda(\lambda-A)^{-1}\right\| \leq M \quad \text { for } \lambda \in \Sigma .
$$

Recall that

$$
d_{k}=\frac{i k\left(\gamma_{0}+b_{k}\right)+\gamma_{\infty}}{c_{0}-a_{k}}, \quad k \in \mathbb{Z} .
$$

Assuming that $\gamma_{0}+b_{k} \neq 0, c_{0}-a_{k} \neq 0, k \in \mathbb{Z}$, we have

$$
\begin{aligned}
M_{k} & =\frac{i k}{c_{0}-a_{k}} R\left(d_{k}, A\right)=\frac{1}{\gamma_{0}+b_{k}}\left(d_{k}-\frac{\gamma_{\infty}}{c_{0}-a_{k}}\right) R\left(d_{k}, A\right) \\
& =\frac{1}{\gamma_{0}+b_{k}} d_{k} R\left(d_{k}, A\right)-\frac{\gamma_{\infty}}{\left(c_{0}-a_{k}\right)\left(\gamma_{0}+b_{k}\right)} R\left(d_{k}, A\right) .
\end{aligned}
$$

On the other hand, we have

$$
\begin{aligned}
2 \Re\left(d_{k}\right) & =d_{k}+\bar{d}_{k} \\
& =\frac{1}{c_{0}-a_{k}}\left(i k\left(\gamma_{0}+b_{k}\right)+\gamma_{\infty}\right)+\frac{1}{c_{0}-\bar{a}_{k}}\left(-i k\left(\gamma_{0}+\bar{b}_{k}\right)+\gamma_{\infty}\right) \\
& =\frac{\gamma_{\infty}}{c_{0}-a_{k}}+\frac{\gamma_{0}}{c_{0}-\bar{a} k}+i k\left(\frac{\gamma_{0}+b_{k}}{c_{0}-a_{k}}-\frac{\gamma_{0}+\bar{b}_{k}}{c_{0}-\bar{a}_{k}}\right) \\
& =\frac{\gamma_{\infty}}{c_{0}-a_{k}}+\frac{\gamma_{0}}{c_{0}-\bar{a}_{k}}+\frac{i k b_{k}}{c_{0}-a_{k}}-\frac{i k \bar{b} k}{c_{0}-\bar{a} k}+\frac{i k \gamma_{0}\left(a_{k}-\bar{a} k\right)}{\left(c_{0}-a_{k}\right)\left(c_{0}-\bar{a} k\right)} .
\end{aligned}
$$

With the hypothesis that the sequences $\left(k a_{k}\right)$ and $\left(k b_{k}\right)$ are bounded, we see that $\left(\Re\left(d_{k}\right)\right)$ is bounded.

With the above estimates, using again the convergences $b_{k} \rightarrow 0, a_{k} \rightarrow 0$ and the estimate 


$$
\begin{aligned}
\left\|M_{k}\right\| & =\left\|\frac{d_{k}}{\gamma_{0}+b_{k}}\left(d_{k}-A\right)^{-1}-\frac{\gamma_{\infty}}{\left(c_{0}-a_{k}\right)\left(\gamma_{0}+b_{k}\right)}\left(d_{k}-A\right)^{-1}\right\| \\
& \leq \frac{1}{\left|\gamma_{0}+b_{k}\right|}\left\|d_{k}\left(d_{k}-A\right)^{-1}\right\|+\frac{\gamma_{\infty}}{\left|\left(c_{0}-a_{k}\right)\left(\gamma_{0}+b_{k}\right)\right|}\left\|\left(d_{k}-A\right)^{-1}\right\|,
\end{aligned}
$$

we see that the sequence $\left\{M_{k}\right\}_{k \in \mathbb{Z}}$ is bounded.

Consider the special case where $a(\cdot) \equiv 0, b(t)=c e^{-\delta t}, t \geq 0$ with $\delta>0$, $c>0, \gamma_{0}>0$ and $\gamma_{\infty}>0$, which was studied by Da Prato and Lunardi [14]. Here $\widetilde{b}(\lambda)=c /(\delta+\lambda)$ for $\Re(\lambda)>-\delta$, and then $b_{k}=c /(i k+\delta), k \in \mathbb{Z}$, and

$$
c_{0} d_{k}=i k\left(\gamma_{0}+b_{k}\right)+\gamma_{\infty}=i k\left(\gamma_{0}+\frac{c \delta}{k^{2}+\delta^{2}}\right)+\frac{c k^{2}}{k^{2}+\delta^{2}}+\gamma_{\infty} .
$$

It follows that

$$
\Re\left(d_{k}\right)=\frac{c k^{2}}{c_{0}\left(k^{2}+c^{2}\right)}+\frac{\gamma_{\infty}}{c_{0}} \geq \gamma_{\infty}>0 .
$$

Hence $\left\{d_{k}\right\}_{k \in \mathbb{Z}} \subset \Sigma$ and

$$
\sup _{k}\left\|d_{k}\left(d_{k}-A\right)^{-1}\right\|<\infty .
$$

We note that $\lim _{|k| \rightarrow \infty}\left|d_{k}\right|=\infty$ since by the Riemann-Lebesgue lemma, $\lim _{|k| \rightarrow \infty}\left|a_{k}\right|=\lim _{|k| \rightarrow \infty}\left|b_{k}\right|=0$. Combining this with the above bound, we obtain

$$
\sup _{k}\left\|\left(d_{k}-A\right)^{-1}\right\|<\infty .
$$

Hence we see that if $A$ generates a bounded holomorphic semigroup, then $\left(M_{k}\right)$ is bounded.

The theorems established in this section recover on the one hand [5, Theorem 2.3], [3, Theorem 4.2] and the maximal regularity result in vectorvalued periodic Besov spaces with periodic boundary conditions in $[6$, Theorem 4.1] which deal with the problem

$$
\left\{\begin{array}{l}
u^{\prime}(t)=A u(t)+f(t), \quad 0 \leq t \leq 2 \pi \\
u(0)=u(2 \pi)
\end{array}\right.
$$

On the other hand, if $a(\cdot) \equiv 0$ in $(2.1)$, we obtain the problem considered by Clément and Da Prato in [11]. They consider solutions on the real line and their main tools are a construction of a "resolvent" for the equation, assuming that $A$ generates an analytic semigroup, and an application of the Da Prato-Grisvard theorem. The results obtained here for the $L^{p}$ case are not attainable by their method (they obtain results on Sobolev spaces instead; however, it is remarkable that they do not impose any geometric condition on the Banach space $X)$. When $b(\cdot) \equiv 0$ in $(2.1)$, the corresponding equation has been considered by Da Prato and Lunardi in [14], using a 
different method, namely the construction of a resolvent for $A$ generating an analytic semigroup. In the latter paper, the authors also treat the case where $a(\cdot)$ is operator-valued, as does J. Prüss in [27] (who studies bounded solutions). Here we drop the assumption that $A$ be a semigroup generator. Equation $(2.1)$ with $b(\cdot) \equiv 0$ was also studied in [20] by the method of operator-valued Fourier multipliers.

Hölder-continuous solutions of the full equation on the real line were studied by Lunardi [23] with the assumption that $A$ is the generator of an analytic semigroup.

In view of the consideration of the semilinear problem in the next section, we single out the following result which is contained in Theorem 3.12. In fact, Hölder spaces are a particular case of the Besov spaces $B_{p, q}^{s}$. Indeed, for $s>0, s \notin \mathbb{N}$, we have $B_{\infty, \infty}^{s}(0,2 \pi ; X)=C^{s}(0,2 \pi ; X)$ (see [6, Theorem 2.1] and [30, Theorem 2.5.7]).

Corollary 3.15. Let $X$ be a Banach space. Assume that the sequences $\left(a_{k}\right)$ and $\left(b_{k}\right)$ satisfy $(\mathrm{H} 2)$ and $\left(a_{k}\right)$ satisfies $(\mathrm{H} 1 \mathrm{~b})$. Then for $0<s<1$ the following statements are equivalent:

(i) $\left\{d_{k}\right\}_{k \in \mathbb{Z}} \subseteq \varrho(A)$ and $\left(M_{k}\right)$ is bounded.

(ii) The problem

$$
\left\{\begin{array}{l}
\frac{d}{d t}\left(\gamma_{0} u(t)+\int_{-\infty}^{t} b(t-s) u(s) d s\right)+\gamma_{\infty} u(t)=A u(t)+f(t), \quad 0 \leq t \leq 2 \pi, \\
u(0)=u(2 \pi) .
\end{array}\right.
$$
is well-posed in $C^{s}(0,2 \pi ; X)$.

Moreover the following maximal regularity property holds:

$$
\left\|u^{\prime}\right\|_{C^{s}}+\|A u\|_{C^{s}}+\|a \dot{*} A u\|_{C^{s}}+\|b \dot{*} u\|_{C^{s}}+\left\|\frac{d}{d t}(b \dot{*} u)\right\|_{C^{s}} \leq C\|f\|_{C^{s}},
$$

where $C>0$ is a constant independent of $f \in C^{s}(0,2 \pi ; X)$.

4. Application to a semilinear problem. In this section, we study solvability in $C^{s}(0,2 \pi ; X), 0<s<1$, for the semilinear problem

$$
\begin{aligned}
\frac{d}{d t}\left(\gamma_{0} u(t)+\right. & \left.\int_{-\infty}^{t} b(t-s) u(s) d s\right)+\gamma_{\infty} u(t) \\
& =c_{0} A u(t)-\int_{-\infty}^{t} a(t-s) A u(s) d s+G(u(t))+\varrho f(t)
\end{aligned}
$$

where $\varrho>0$ is a small parameter.

In the case $a(\cdot) \equiv 0$ and $\varrho=1$ this problem was studied on the real line by Clément and Da Prato [11] and Sforza [28] considered solutions in the 
large. A model for this equation is the problem of nonlinear heat conduction in materials of fading memory type, where memory is also present in the thermal conductivity term of the right-hand side:

$$
\begin{aligned}
\frac{d}{d t}\left(\gamma_{0} u(t, x)+\int_{-\infty}^{t} b(t-s) u(s, x) d s\right)+\gamma_{\infty} u(t) & \\
= & c_{0} \Delta u(t, x)-\int_{-\infty}^{t} a(t-s) A u(s) d s \\
& \quad+g(x, u(t, x), \nabla u(t, x))+f(t, x), \quad x \in \Omega .
\end{aligned}
$$

Here, $\Omega \subset \mathbb{R}^{n}$ is open and bounded, and

$$
\Delta=\sum_{j=1}^{n} \frac{\partial}{\partial x_{j}^{2}}
$$

is the Laplace operator with Dirichlet boundary conditions on $X=C(\bar{\Omega})$. The positive constants $\gamma_{0}$ and $c_{0}$ represent the heat capacity and the thermal conductivity, respectively, for the material under study (see [28], [13] and [25]).

Theorem 4.1. Let $X$ be a Banach space and $0<s<1$. Assume that the sequences $\left(a_{k}\right)$ and $\left(b_{k}\right)$ satisfy $(\mathrm{H} 2)$ and $\left(a_{k}\right)$ satisfies $(\mathrm{H} 1 \mathrm{~b})$. Suppose that

(i) $\left\{\frac{i k\left(\gamma_{0}+b_{k}\right)+\gamma_{\infty}}{c_{0}-a_{k}}\right\} \subseteq \varrho(A)$ and $\left\{\frac{i k}{c_{0}-a_{k}}\left(\frac{i k\left(\gamma_{0}+b_{k}\right)+\gamma_{\infty}}{c_{0}-a_{k}} I-A\right)^{-1}\right\}$ is bounded.

(ii) $G(0)=0 ; G$ is continuously (Fréchet) differentiable at $u=0$ and $G^{\prime}(0)=0$.

Then there exists $\varrho^{*}>0$ such that equation (4.1) is solvable for each $\varrho \in$ $\left[0, \varrho^{*}\right)$, with solution $u=u_{\varrho} \in C^{s}(0,2 \pi ; X)$.

Proof. Define the operator $L_{0}$ by

$$
\begin{aligned}
D\left(L_{0}\right)= & C^{1+s}(0,2 \pi ; X) \cap C^{s}(0,2 \pi ; D(A)) \\
\left(L_{0} u\right)(t)= & \frac{d}{d t}\left(\gamma_{0} u(t)+\int_{-\infty}^{t} b(t-s) u(s) d s\right) \\
& +\gamma_{\infty} u(t)-c_{0} A u(t)+\int_{-\infty}^{t} a(t-s) A u(s) d s
\end{aligned}
$$

where $D(A)$ is endowed with the graph norm. We consider, for $\varrho \in(0,1)$, the one-parameter family of problems

$$
H[u, \varrho]=-L_{0} u+G(u)+\varrho f .
$$


Keeping in mind that $G(0)=0$, we see that $H[0,0]=0$. Also, by hypothesis, $H$ is continuously differentiable at $(0,0)$ and, by Remark 2.10 , for $s \in(0,1)$ fixed the operator $L_{0}$ is an isomorphism onto. Hence the partial Fréchet derivative $H_{(0,0)}^{1}=L_{0}$ is invertible. The conclusion of the theorem now follows from the implicit function theorem (see e.g. [17, Theorem 17.6]).

REMARK 4.2. Under additional conditions on the nonlinearity $G$, it is possible to remove the rather restrictive hypothesis $G^{\prime}(0)=0$. In fact, in this case all that we need is that the linear mapping

$$
H_{(0,0)}^{1}=L_{0}+G^{\prime}(0)
$$

be invertible. In case $a \equiv 0$ for (4.1), this is contained in [28, Proposition 3.2 ], requiring the following condition:

$\gamma_{0}>0, \gamma_{\infty}>0$, and $b(\cdot):[0, \infty) \rightarrow \mathbb{R}$ is nonnegative and nonincreasing.

About the nonlinearity $G$, we can make the following assumptions as in [28]:

(H4) There exists a subspace $X_{\alpha}, 0 \leq \alpha<1$, such that $X_{\alpha}$ is a Banach space with norm $\|\cdot\|_{\alpha}, G: X_{\alpha} \rightarrow X$ and:

(1) $D(A) \subset X_{\alpha} \subset X$ with continuous embeddings;

(2) there exists $c_{\alpha}>0$ such that for all $x \in D(A)$,

$$
\|x\|_{X_{\alpha}} \leq c_{\alpha}\|x\|_{D(A)}^{\alpha}\|x\|^{1-\alpha}
$$

(3) $G(0)=0$ and $G$ is locally Lipschitz-continuous;

(4) $G$ is differentiable in $X_{\alpha}$ and $G^{\prime}$ is locally Lipschitz-continuous.

The final assumption we make is that $A+G$ is dissipative in the sense that

$$
\|x-y\| \leq\|x-y-\lambda(A(x-y)+G(x)-G(y))\| \text { for any } x, y \in D(A)
$$
and $\lambda>0$.

As seen by adapting the proofs of [28], under these assumptions, an analog to Theorem 4.1 holds for problem (4.1) even with $\varrho=1$ but in case $a \equiv 0$ holds. The details are left to the reader.

Remark 4.3. Compared with [11] and [28], we consider here the periodic problem and do not impose the condition that $A$ be the generator of an analytic semigroup.

REMARK 4.4. We remark that the $R$-boundedness assumption in Theorem 3.9 is satisfied by a large number of examples. We refer to the recent monographs by Denk, Hieber and Prüss [16] and Kunstmann and Weiss [21] for the corresponding developments. The authors thank the referee for pointing out the above references to them. 


\section{References}

[1] H. Amann, Linear and Quasilinear Parabolic Problems, Monogr. Math. 89, Birkhäuser, Basel, 1995.

[2] - On the strong solvability of the Navier-Stokes equations, J. Math. Fluid Mech. 2 (2000), 16-98.

[3] W. Arendt, C. Batty and S. Bu, Fourier multipliers for Hölder continuous functions and maximal regularity, Studia Math. 160 (2004), 23-51.

[4] W. Arendt, C. J. K. Batty, M. Hieber and F. Neubrander, Vector-Valued Laplace Transforms and Cauchy Problems, Monogr. Math. 96, Birkhäuser, Basel, 2001.

[5] W. Arendt and $\mathrm{S}$. Bu, The operator-valued Marcinkiewicz multiplier theorem and maximal regularity, Math. Z. 240 (2002), 311-343.

[6] —, - Operator-valued Fourier multipliers on periodic Besov spaces and applications, Proc. Edinb. Math. Soc. (2) 47 (2004), 15-33.

[7] J. Bourgain, Vector-valued singular integrals and the $H^{1}-B M O$ duality, in: Probability Theory and Harmonic Analysis (Cleveland, OH, 1983), Monogr. Festbooks Pure Appl. Math. 98, Dekker, New York, 1986, 1-19.

[8] -, Vector-valued Hausdorff-Young inequalities and applications, in: Geometric Aspects of Functional Analysis (1986/1987), Lecture Notes in Math. 1317, Springer, Berlin, 1988, 239-249.

[9] H. Brézis, Analyse Fonctionnelle. Théorie et Applications, Masson, Paris, 1983.

[10] P. L. Butzer and H. Berens, Semi-Groups of Operators and Approximation, Grundlehren Math. Wiss. 145, Springer, New, York, 1967.

[11] Ph. Clément and G. Da Prato, Existence and regularity results for an integral equation with infinite delay in a Banach space, Integral Equations Operator Theory 11 (1988), 480-500.

[12] Ph. Clément, B. de Pagter, F. A. Sukochev and M. Witvliet, Schauder decompositions and multiplier theorems, Studia Math. 138 (2000), 135-163.

[13] B. D. Coleman and M. E. Gurtin, Equipresence and constitutive equations for rigid heat conductors, Z. Angew. Math. Phys. 18 (1967), 199-208.

[14] G. Da Prato and A. Lunardi, Periodic solutions for linear integrodifferential equations with infinite delay in Banach spaces, in: Differential Equations in Banach Spaces (Bologna, 1985), Lecture Notes in Math. 1223, Springer, Berlin, 1986, 49-60.

[15] - Solvability on the real line of a class of linear Volterra integrodifferential equations of parabolic type, Ann. Mat. Pura Appl. (4) 150 (1988), 67-117.

[16] R. Denk, M. Hieber and J. Prüss, R-boundedness, Fourier multipliers and problems of elliptic and parabolic type, Mem. Amer. Math. Soc. 166 (2003), no. 788.

[17] D. Gilbarg and N. S. Trudinger, Elliptic Partial Differential Equations of Second Order, Grundlehren Math. Wiss. 224, Springer, Berlin, 2nd ed., 1983.

[18] M. Girardi and L. Weis, Operator-valued Fourier multiplier theorems on Besov spaces, Math. Nachr. 251 (2003), 34-51.

[19] - - - Operator-valued Fourier multiplier theorems on $L_{p}(X)$ and geometry of $B a-$ nach spaces, J. Funct. Anal. 204 (2003), 320-354.

[20] V. Keyantuo and C. Lizama, Fourier multipliers and integro-differential equations in Banach spaces, J. London Math. Soc., (2) 69 (2004), 737-750.

[21] P. C. Kunstmann and L. Weis, Maximal $L_{p}$-regularity for parabolic equations, Fourier multiplier theorems and $H^{\infty}$-functional calculus, in: M. Iannelli, R. Nagel and S. Piazzera (eds.), Functional Analytic Methods for Evolution Equations, Lecture Notes Math. 1855, Springer, Berlin, 2004, 65-311. 
[22] S. Kwapień, Isomorphic characterizations of inner product spaces by orthogonal series with vector valued coefficients, Studia Math. 44 (1972), 583-595.

[23] A. Lunardi, The heat equation with fading memory, SIAM J. Math. Anal. 21 (1990), $1213-1224$.

[24] S. Monniaux, On uniqueness for the Navier-Stokes system in 3D-bounded Lipschitz domains, J. Funct. Anal. 195 (2002), 1-11.

[25] J. W. Nunziato, On heat conduction in materials with memory, Quart. Appl. Math. 29 (1971), 187-204.

[26] J. Prüss, Evolutionary Integral Equations and Applications, Monogr. Math. 87, Birkhäuser, Basel, 1993.

[27] —, Bounded solutions of Volterra equations, SIAM J. Math. Anal. 19 (1988), 133149.

[28] D. Sforza, Existence in the large for a semilinear integrodifferential equation with infinite delay, J. Differential Equations 120 (1995), 289-303.

[29] E. M. Stein and G. Weiss, Introduction to Fourier Analysis on Euclidean Spaces, Princeton Math. Ser. 32, Princeton Univ. Press, Princeton, NJ, 1971.

[30] H. Triebel, Theory of Function Spaces, Monogr. Math. 78, Birkhäuser, Basel, 1983.

[31] L. Weis, Operator-valued Fourier multiplier theorems and maximal $L_{p}$-regularity, Math. Ann. 319 (2001), 735-758.

[32] -, A new approach to maximal $L_{p}$-regularity, in: Evolution Equations and Their Applications in Physical and Life Sciences (Bad Herrenalb, 1998), Lecture Notes in Pure and Appl. Math. 215, Dekker, New York, 2001, 195-214.

Department of Mathematics

Faculty of Natural Sciences

University of Puerto Rico

P.O. Box 23355

Puerto Rico 00931, U.S.A.

E-mail: keyantuo@uprrp.edu
Departamento de Matemática Facultad de Ciencias Universidad de Santiago de Chile Casilla 307, Correo 2 Santiago, Chile E-mail: clizama@usach.cl

Received April 5, 2004

Revised version December 14, 2004 